\title{
Management of Chronic Musculoskeletal Pain in the Elderly: Dilemmas and Remedies
}

\author{
Ayse Ozcan Edeer and Hulya Tuna \\ Additional information is available at the end of the chapter
}

http://dx.doi.org/10.5772/50780

\section{Introduction}

Aging is perhaps better understood as a process of gradual change from birth to death, since there is no universally accepted definition of old age. Projections show dramatic increases in older population; approximately by 2030 there will be an estimated 8 million people who are 85 years or older [1-3]. Moreover, $25 \%$ of the population in USA will be age 65 years or older in 2050. The percentage over the age of 85 is expected to triple [4-7]. Europe has experienced the similar transition to an older population profile over the last century which reflects a world-wide demographic trend towards an ageing population. Department of Health reports that in Britain, the number of people aged over 65 years has doubled in the last 70 years and the number of people over 90 years is expected to double in the next 25 years [8]. Similarly D'Astolfo et al state that older adults aged 65 plus, are the fastest growing segment of the Canadian population [9].

The European Union has identified the provision of health and social care for this population as a crucial challenge for the $21^{\text {st }}$ century. In a shift away from merely extending life, ways of reducing morbidity and coping with disability, preventing incapacity, extending the quality of life and enhancing the functional independence of older people will be an important component of service provision $[10,11]$. Recent efforts have begun to concentrate on the predictors of successful aging, but age-based comparisons of the pain experience remain challenging due to the complexity and non-uniformity of the aging process [12]. Therefore, there is an urgent and growing need for interventions that are effective in decreasing pain, suffering, and pain-related disability in this group.

\section{Prevalence of pain in older adults}

Although chronic pain is a highly prevalent and often disabling condition among older adults, the prevalence in the elderly is not properly defined. Some studies suggest that fifty 
percent of community dwelling adults aged 60 years or above have been found to experience pain and this number increases to $45-80 \%$ in the nursing home population with analgesics being used in $40 \%$ to $50 \%$ of residents [1,13-19]. Brown et al report higher percentage and state that more than $90 \%$ of the elderly living in the community experienced pain within the past month [6]. Given the prevalence of chronic pain, its impact on health, and its costs, which approach $\$ 100$ billion annually, chronic pain represents a major public health issue [20].

While the existence of acute pain remains approximately the same across the adult life span, there is an age-related increase in the prevalence of chronic pain at least until the seventh decade of life $[13,15]$. Approximately $57 \%$ of older adults report experiencing pain for 1 or more years compared with less than $45 \%$ of younger people. Furthermore, long-term care data indicate that over $40 \%$ of patients, who were known to have pain at an initial assessment, had worsening or severe pain at the time of the second assessment 2-6 months later [21].

\section{Chronic musculoskeletal pain}

Chronic musculoskeletal pain (CMP) is the most common, non-malignant disabling condition that affects at least one in four older people [22,23]. The most musculoskeletal pain in the joints of the upper and lower extremities, especially hips, knees, and hands, is associated with the degenerative changes of osteoarthritis. Older adults may also develop tendonitis and bursitis, as well as inflammatory joint and muscle disease [24]. The most common painful musculoskeletal conditions among older adults are osteoarthritis, low back pain, fibromyalgia, chronic shoulder pain, knee pain, myofascial pain syndrome and previous fracture sites $[7,23,25]$.

It is reported that the most common causes of pain identified in nursing home patients included arthritis and previous fractures. Arthritis alone affects well over 20 million Americans with an increase to 40 million expected by 2020. Twenty-nine percent of Medicare patients in nursing homes with a fracture in the prior 6 months suffer with daily pain [13]. Also surgical procedures are more frequently performed on older people. In the Medicare population in the United States for example, rates of total joint replacement surgery for patients with severe hip or knee osteoarthritis are more than doubled between 1988 and 1997. Over the same time period, rates of spine surgery in Medicare patients increased by $57 \%$ [23]. Chronic low back pain (CLBP) is one of the most common, poorly understood, and potentially disabling chronic pain conditions in older adults [26]. Many older adults remain quite functional despite CLBP, and because age-related co-morbidities often exist independently of pain, the unique impact of CLBP is unknown [27]. The Framingham Study (1992-1993) reported $63 \%$ of women pain in one or more regions, compared to $52 \%$ of men. Widespread CMP was more prevalent among women than men (15 versus $5 \%$, respectively) [28].

Finding that CMP is linked with the subsequent development of severe mobility disability may have important public health implications for the rapidly aging population [29]. Among 898 nondisabled community-dwelling older adults, it was found that the risk of disability increases with the number of areas reported with CMP [30]. The results of the another study indicated that more than $90 \%$ of the elderly living in the community experienced pain within 
the past month, with $41 \%$ reporting discomforting, distressing, unbearable, or severe pain. CMP was found to be the most predominant pain, and inactivity was the most effective strategy used to lessen pain [6]. D'Astolfo et al emphasized that CMP is a significant burden on the Canadian health care system. It is considered the third most expensive disorder in terms of spent health care dollars, surpassed only by cancer and heart disease [9].

\section{Consequences of chronic musculoskeletal pain}

The impact of CMP is a cycle of disuse and inactivity. This cycle in turn leads to a further reduction in function, accompanying psychological effects and decreased quality of life [7,31]. Interrelated problems caused by the inadequate treatment of pain in older adults have been highlighted by several authors. Consequences of poorly managed CMP in this population may include fear of movement, decreased ambulation, functional decline, functional dependence, disability, impaired posture, risk of pressure sores, muscle atrophy, increased subsequent exacerbation of frailty. Older adults may also have impaired appetite, malnutrition, impairment of excretory functions (bowel and bladder) and impaired memory, the impairment of enjoyable recreational activities, impaired dressing and grooming, sleep disturbance, behavioral problems, social isolation, depression, anxiety and even suicidal thoughts [7,10,14-16]. Furthermore, depression, behavioral changes, and cognitive impairment can complicate therapy and make assessment more difficult. Paininduced decline in mobility and activity may further lead to increase the risk of trauma, particularly caused from falls [14].

Falls are one of the major causes of death among older adults and the most important cause of hospitalization and increased healthcare utilization and costs in this population [9]. CMP measured according to number of locations, severity, or pain interference with daily activities is associated with greater risk of falls in older adults [32-35]. Leveille et al conducted the population-based study. At baseline, $40 \%$ of participants reported polyarticular chronic pain, and another $24 \%$ reported chronic pain in only one joint area. A total of 1,029 falls were reported during 18 months of follow-up. The researchers found that patients who had chronic pain had higher rates of falls during follow-up than those who were pain-free [33]. In the another population based study, total of 605 participants aged 75 years and older, CMP was reported by $48 \%$ of the participants, of whom majority had moderate to severe pain in lower extremities or back. The participants with moderate to severe pain had more than twice (odds ratio 2.33, 95\% confidence interval 1.44-3.76) the risk for impaired balance compared with those without pain. The researchers came up with a conclusion that there was a direct relationship between the moderate to severe CMP and impaired postural balance [36].

\section{Ineffective management of chronic musculoskeletal pain in older adults}

In spite of high prevalence and consequences of CMP among older adults, there have been relatively few studies in older populations with pain. Studies have indicated that less than 
$1 \%$ of the thousands of papers published on pain focus on the aging society $[13,17]$. Therefore, health care professionals remain ineffective in assessing and treating pain. Improving the health care professionals' knowledge and skills related to pain assessment in older adults and adopting aggressive approaches to comprehensive pain assessment are crucial to improve older adult's quality of life [15,37]. The study conducted recently reported that although CLBP was a common and debilitating problem in older adults, primary care physicians did not feel "very confident" in their ability to diagnose any of the contributors of CLBP listed (most items $<40 \%$ ). The results point to a need for more primary care physician education about CLBP in older adults [38].

Older people may not report pain, and nurses or caregivers may not enquire about it. Both older people and their caregivers can hold age related attitudes regarding pain and view pain as an expected consequence of the ageing process. Older adults may not report pain because they do not want to be a burden for their families and caregivers. It results mostly in lack of information by healthcare professionals about pain control of older people. Furthermore, extensive documentation requirement may deter health-care professionals from appropriately prescribing effective treatments [39,40]. Other factors such as inadequate reimbursement and financial incentives for pain management efforts, negative reinforcement in training programs for attending to pain while being rewarded for less important and more detailed interventions, lack of training for pain management skills, lack of recognition and interaction among various medical disciplines (and even among different pain groups), limited access to diagnostic or therapeutic facilities or experts, inadequate pharmacy services, insufficient staffing for proper pain assessment and interventions, inflexible access to medications based on formulary selections, and other restrictive policies may also contribute to failure in treatment of pain $[4,40]$.

\section{Management of chronic musculoskeletal pain in older adults}

Treatments for CMP are focused at decreasing pain, making it more tolerable and improving patients function. Considering the needs of individual older patients can better explain what their expectations are regarding pain treatment outcomes. Treating pain should be done individually as well as following some general principles. Multidisciplinary pain programs that combine several modes of pharmacological and non-pharmacological treatment have demonstrated efficacy for the management of chronic pain in older adults. However, those programs appear to be not being used effectively, because older patients are less likely to be offered this treatment in pain management clinics, and receive fewer treatment options when attending such clinics due to inadequate representation $[12,41]$.

Pharmacological therapy for chronic musculoskeletal pain is the most effective when combined with non-pharmacological approaches: physical therapy (e.g., exercise program, TENS, application of heat or cold), psychological methods (e.g., relaxation, biofeedback, hypnosis, cognitive-behavioral therapy), educational programs, social interventions and complementary therapies (e.g., acupuncture) [14,16,37,41,]. In an older population, where the risk of adverse events is higher, the non-pharmacologic options will usually cost less and cause fewer side effects. 


\section{Pharmacological therapy}

Although the high risk for adverse drug reactions in the older adults, pharmacologic interventions remain the primary modality for treating CMP in the geriatric population [20]. The management of CMP in older patients mostly consists of opioids, non-opioids and adjuvant analgesics.

Drug distribution usually is different in older patients as compared to younger patients because of changes in blood flow to organs, protein binding, and body composition that occur with aging [3]. In addition, many older adults continue to report substantial pain despite the regular use of analgesic medications. Polypharmacy, as well as inappropriate prescribing, for the older patients is a major problem and a challenge that contributes to costs, adverse drug events, confusion, compliance issues, and errors in management. [42-44] It is reported that CMP is one of the most common geriatric consultation and admission the hospital. Geriatric consultations increase the total number of medications and the cost of medications used by elderly patients. These restrictions have led to a need for effective nonpharmacological interventions to manage CMP [42].

\subsection{Nonopioids}

According to American Geriatric Society (AGS) nonopioids are generally the first line of therapy for mild to moderate or "tolerable" CMP [16,23,43]. Acetaminophen (APAP) and nonsteroidal anti-inflammatory drugs (NSAIDs) are among the most common analgesics used to. APAP is usually the first choice because it is relatively safe for older people. It was reported that APAP treatment reduced pain behaviors associated with musculoskeletal pain in persons with dementia in community-dwelling [45,46]. Dosing of APAP should be limited to avoid liver toxicity, and topical analgesics are preferred for focal pain. The longterm use of NSAIDs should be avoided when possible because of their high frequency of adverse effects; e.g., risks of gastrointestinal bleeding and renal dysfunction which are significantly higher in older adults than in the younger population. The newer cyclooxygenese-2 (COX)-2 inhibitor NSAIDs are believed to be associated with a lower side effect profile in older adults [41]. COX-2 inhibitor NSAIDs has been linked to an increased incidence of acute coronary syndrome, although there is evidence that cardiovascular-related adverse events are not limited to the selective COX-2 inhibitors. Additionally, chronic use of either APAP or NSAIDs has been associated to elevations in blood pressure [47].

Data was collected from 428 patients aged $\geq 50$ years with non-inflammatory musculoskeletal pain during a consultation with their general practitioner (GP). In cases, where a prescription is issued, this is more strongly influenced by previous NSAID prescriptions than the patient's pain level. Researchers concluded that GPs mostly adopt an individualized approach to the treatment of musculoskeletal pain in older adults [48]. A survey of inpatients' drug knowledge showed that $66-90 \%$ of older adults did not know which of several medicines contained APAP, and only $7 \%$ knew the maximum daily dose. Therefore close monitoring of pain medication use is necessary in older patients, particularly those with cognitive impairment. According to the 2009 guidelines for 
pharmacologic management of persistent pain in older persons published by the AGS, NSAIDs should be used only with extreme caution in highly selected individuals once other safer therapies have failed. Absolute contraindications for NSAIDs use in older adults are chronic kidney disease, heart failure, and active peptic ulcer disease $[16,46,49]$.

\subsection{Opioids}

Worries connected to taking opioids and a reluctance to report pain have caused inefficient pain management with opioids in older patients. In reality, addiction risk with opioids is low $(<0.1 \%)$ when analgesics are used for acute pain in patients who are not substance abusers [4]. Opioids are one of the pharmacologic classes recommended for treatment from moderate to severe pain in guidelines released in 2009 by the AGS. According to the AGS, opioids should be considered for patients who have pain related functional impairment or diminished quality of life due to pain [23,50].

Within this population, short-acting opioids can be used in treatment of patients with intermittent pain, whereas sustained-release opioids should be given for continuous pain (with short-acting preparations available for breakthrough pain). Once total daily dose requirements have been determined, a long acting agent may be used. Sustained-released opioids should be used for the treatment of continuous pain while using short-acting preparations for breakthrough pain. Both morphine and oxycodone are commonly used and available in both short-acting and sustained-release preparations. For patients who may not be able to take oral preparations periodically, opioids are available as parenteral, sublingual (buprenorphine hydrochloride), suppository (oxymorphone hydrochloride), and transdermal (eg, fentanyl patch) products. Long-acting opioids should seldom be initiated in opioid-naive older patients [7,46].

Patient-controlled analgesia (PCA), whether using oral or parenteral agents, can be most beneficial in a cognitively intact population, with the likelihood of the best pain control in conjunction with the least amount of opioid needed to control musculoskeletal pain [4].

An oral long-acting agent such as morphine (the oral dose required is usually about 3 to 4 times greater than the parenteral dose needed for the same duration) or oxycontin in conjunction with a similar short-acting agent can also be used. Some people will metabolize the medication more quickly, and if breakthrough pain occurs after 8 hours of adequate pain relief, therefore the solution would be to increase the frequency of dosing to every 8 hours from every 12 hours rather than to increase the 12-hour dosage. A controlled-release morphine or controlled-release oxycodone should never be prescribed more frequently than every 8 hours [4].

Meperidine hydrochloride should not be used because of the accumulation of a nephrotoxic metabolite. Benzodiazepines have also been used in the treatment of a variety of painful conditions, particularly muscle spasms related to pain crises. Transdermal fentanyl patches should generally be avoided as a first-line agent in older patients, because absorption is unpredictable, being affected by differences in body temperature and subcutaneous fat and 
water in older patients as compared with younger adults studied in clinical trials [23]. Trescot et al reported that long-term effectiveness of 6 months or longer use of opioids is variable with evidence ranging from moderate for transdermal fentanyl and sustainedrelease morphine with a Level II-2, to limited for oxycodone with a Level II-3, and indeterminate for hydrocodone and methadone with a Level III [51].

Although opioid therapies may have a lower risk for organ failure than other therapies, confusion, dizziness, nausea, sedation, constipation, impaired balance, falls and hip fractures, depression, and agitation are other potential related side effects that can affect this population in particular. Finally, older adults with CMP taking opioid analgesics should be reassessed for ongoing attainment of therapeutic goals, adverse effects, and safe and responsible medication use [46].

\subsection{Adjuvant analgesics}

Adjuvant medications, while not classically categorized as analgesics, may be effective in treating certain CMP syndromes in older adults. Steroids, anticonvulsants, topical local anesthetics, and antidepressants are adjuvant agents. Depression/anxiety is often unnoticed in older patients and requires consideration when managing patients with pain $[4,23,41]$. The study of the relationship between depression and pain complaints in older patients has revealed that initial control of depression greatly facilitates pain management. If depression is not addressed aggressively, interventions to manage pain are unlikely to be successful [7]. Tertiary amines (e.g., amitriptyline, imipramine, trimipramine, doxepin, clomipramine, should be avoided in older patients because of greater anticholinergic side effects, including sedation, delirium, urinary retention, constipation, glaucoma exacerbation, and dizziness and, for amitriptyline, especially, the risk of cardiac arrhythmia. By contrast, secondary amines (nortriptyline, desipramine, protriptyline, amoxapine) tend to have better adverse event profiles in older patients [41].

Tramadol is another agent available to help control mild to moderate pain and, except in a substance-abuse population. It should have a low tolerance problem and may be beneficial in a variety of pain situations [23].

In about $90 \%$ of cases, additional adjuvant medications will be needed to control pain. Vitamin D is also likely to be helpful in some pain situations. Vitamin D and calcium have also been shown to decrease fracture rates, which are a source of pain themselves. Lower concentrations of $25(\mathrm{OH}) \mathrm{D}$ are associated with significant back pain in older women, but not men. Because vitamin D deficiency and CMP are fairly prevalent in older adults, these findings suggest it may be worthwhile to query older adults about their pain and screen older women with significant back pain for vitamin D deficiency [52]. Calcitonin has been shown in clinical trials to relieve pain associated with vertebral compression fractures. Topical agents are also available for site-specific pain. Also, topical treatments can be useful for patients who have difficulty swallowing pills and for patients taking multiple medications. The safety of topical lidocaine has been established as well. Topical capsaicin should be started at the lowest dose recommended. However the burning sensation 
associated with capsaicin application during the chemical desensitization phase makes for poor tolerability; many older patients are not able to endure the treatment long enough to achieve therapeutic effects $[4,41,50]$.

Adverse drug reactions occur more than twice as frequently among older adults than younger ones and increase as the number of medications increases. On average, a 70-yearold takes seven different medications. A high prevalence of medication errors in older adults results from accumulation of factors that contribute to medication errors in all age groups, such as polypharmacy, polymorbidity, enrollment in several disease-management programs, and fragmentation of care [53]. The essential approach to treating older adults is not necessarily to find a set number of medications and try to stay below it, but to find the right medication at the right dosage and for the shortest possible duration on a case-by-case basis. This individualized approach to treating patients will provide a much safer and more effective means of practicing and will improve patients' quality of life [54]. In general, as specific initial and titrating dosage regimens for the elderly are not readily available, the "start low and go slow" approach to drug prescribing in the elderly is particularly important as it applies to pain management (AGS 2002) [16,21,42].

\section{Non-pharmacological approaches}

\subsection{Physical therapy}

Ideally, first-line interventions should directly address the source of pain in older adults with CMP. A comprehensive examination of the patient to identify impairments associated with the painful condition will direct those interventions. Physical therapy interventions reduce stress and correct malalignments of joint structures, correct muscle imbalances, and enhance the shock absorption capacity of tissue structures. Selection of appropriate treatments must include consideration of contraindications associated with the patient's comorbid conditions (e.g., osteoporosis or osteopenia) [10,20,57].

Passive treatment modalities focused solely on temporarily decreasing pain symptoms (e.g., heat treatments, cryotherapy, transcutaneous electrical nerve stimulation [TENS]) should be used sparingly as part of the physical therapy intervention [1,2]. These modalities should be a means to an end, the end being decreasing pain to a sufficient extent to allow patients to participate in subsequent active treatments aimed at positively affecting functional abilities [20,50,55-57].

\subsubsection{Thermal agents}

Superficial heating agents (e.g. hot packs, warm hydrotherapy, paraffin, fluidotherapy and infrared) or deep heating agents (e.g. short-wave and microwave diathermy, and ultrasound) can be used to increase blood flow, membrane permeability, tissue extensibility and joint range of motion in ways that can contribute to decreasing pain. Heat and cold alter both peripheral and central nervous system excitability, and can thus serve as a means of modulating pain [20,58]. 
Although thermal agents are frequently used in the physical therapy treatment of patients with pain, the literature on the effects of thermal agents on pain in older adults is limited. Thermal agents are commonly used in the self-management of chronic pain [55]. In a study of 235 (mean age of 82 years) community-dwelling adults, Acetaminophen, regular exercise, prayer, and heat and cold were the most frequently used pain management strategies $(61 \%$, $58 \%, 53 \%$, and $48 \%$, respectively). 272 community-dwelling older adults aged 73 years or older reported hot and/or cold modalities (28\%) as a pain-reduction strategy [59]. Chatap et al conducted a study to determine the effects of hyperbaric $\mathrm{CO}(2)$ cryotherapy in older adults with pain whose origin was usually musculoskeletal $(80.3 \%)$. They found that the pain scores decreased significantly after four sessions, from $45 \mathrm{~mm}$ to $13 \mathrm{~mm}$ on visual analog scale $(\mathrm{P}<0.001)$ in those with chronic pain. They concluded that hyperbaric $\mathrm{CO}(2)$ cryotherapy is an innovative tool that should be incorporated within the nonpharmacological armamentarium for achieving pain relief in older patients [60].

\subsubsection{Manual therapy}

Although there is scant evidence on the use of joint mobilization and manipulation specifically for older adults, research has addressed the use of these treatments for knee and hip osteoarthritis (OA), conditions common in older adults [20]. A recent qualitative systematic review aimed to determine if manual therapy improves pain and/or physical function in people with hip or knee OA. Four RCTs were eligible for inclusion (280 subjects), three of which studied people with knee OA and one studied those with hip OA. There is silver level evidence that manual therapy is more effective than exercise for those with hip $\mathrm{OA}$ in the short and long-term. The researchers concluded that due to the small number of RCTs and patients, this evidence could be considered to be inconclusive regarding the benefit of manual therapy on pain and function for knee or hip OA [61].

A Cochrane systematic review concludes that manual therapy alone is insufficient in the management of persistent neck pain. However, there is strong evidence that either manipulation or mobilization combined with exercise is effective in reducing pain. This review also concluded that manual therapy with exercise improves function and the patients' global perceived effect of treatment [62]. The Philadelphia Panel (2001) concluded that there were insufficient data for the general population to reach a conclusion about the effect of massage for low back pain, neck pain, and shoulder pain. A systematic review by Harris et al [63], determined that slow-stroke back massage and hand massage showed statistically significant improvements on physiological or psychological indicators of relaxation in older people. A limited number of studies on massage have been conducted exclusively with older individuals. Hawk et al compared the clinical outcomes of spinal manipulation and a non-manipulative mind-body approach (Bioenergetic Synchronization Technique) for patients with chronic musculoskeletal pain in older adults. They reported that for this particular group of patients, both groups demonstrated similar improvement scores on the Pain Disability Index [64]. 


\subsubsection{Protective and supportive devices}

Protective and supportive devices assist a decrease in pain and increase in function for patients with joint instability or malalignment. Therapeutic taping for patellar realignment is effective in reducing pain and improving function in patients with osteoarthritis of the knee. Recently introduced kinesiotaping method helps to increase blood circulation, decrease pain and relaxation on fascia, tendon and muscles regarding painful musculoskeletal conditions. Impact-absorbing shoes may help to relieve foot, ankle, knee and hip pain from osteoarthritis. Patients with metatarsalgia associated with rheumatoid arthritis experienced decreased pain using custom-fitted foot orthotics. Besides supportive and protective devices, ambulation devices like wheelchair, cane, crutch etc. can help to relieve stress from lower extremity especially during immobilization period after musculoskeletal injuries in older adult. Therefore appropriate device selection and measurements are important in order to improve efficiency. Decisions regarding the use of protective or supportive devices should therefore be individualized to the patient based on the information gained in the examination $[20,57,58]$.

\subsubsection{Transcutaneous electrical nerve stimulation}

Despite positive conclusions regarding the use of transcutaneous electrical nerve stimulation (TENS), methodological weaknesses of published studies limit the ability to conclusively support the use of TENS for chronic pain conditions in older adults. High-frequency TENS appears to be the most effective TENS application for postsurgical pain and can be used with modulating frequencies to control neurologic accommodation. A recent systematic review of TENS for persistent pain concluded that an insufficient number of high-quality randomized clinical trials existed to evaluate the use of TENS for the management of persistent pain. To date, only a small number of studies have been found that examined the effect of TENS exclusively with older adults $[57,58]$.

Most recently, van Middelkoop $\mathrm{M}$ et al found no difference in effectiveness of TENS and sham TENS and no difference between TENS and active treatments. The data provided low quality evidence for TENS versus sham-TENS and very low quality evidence that percutaneous electrical nerve stimulation (PENS)/acupuncture is more effective than TENS for post-treatment and short-term pain relief [65]. They concluded that application of TENS attenuates blood pressure and vasoconstrictor responses during exercise and metaboreflex activation, associated with improved sympatho-vagal balance in healthy young and older individuals [66]. A recent study by Weiner et al provides some support for the use of percutaneous electrical nerve stimulation (PENS) for low back pain in older adults. Subjects randomized to PENS plus physical therapy intervention had significantly greater reductions in pain intensity measures at the end of the 6 weeks $(\mathrm{P}<.001)$. These pain reduction effects were maintained at 3-month follow-up [67].

In evidence-based meta analysis by Zhang $W$ et al in 2008, authors search recommendations for the management of hip and knee osteoarthritis (OA). Recommendations cover the use of 12 non-pharmacological modalities: education and self-management, regular telephone 
contact, referral to a physical therapist, aerobic, muscle strengthening and water-based exercises, weight reduction, walking aids, knee braces, footwear and insoles, thermal modalities, TENS and acupuncture [68]. Same author groups made similar meta-analysis in 2010 and reported that among non-pharmacological therapies, effect size for pain relief was unchanged for self-management, education, exercise and acupuncture. However, with new evidence the effect size for pain relief for weight reduction reached statistical significance [69].

\subsection{Complementary and alternative therapies}

Complementary and alternative medicine is most often used to treat painful musculoskeletal conditions as well as conditions that are comorbid with pain in older adults as a holistic therapy. [70]. Molton et al researched the pain coping strategies among older, middle-aged, and younger adults living with CMP. They reported older adults report a wider range of frequently used strategies and significantly more frequent engagement in activity pacing, seeking social support, and use of coping self-statements than did younger or middle-aged adults [71]. Self-management programs for pain have particular relevance for the field of geriatric pain management [56,71]. Despite their documented efficacy in young to middle aged samples cognitive-behavioral and self-management pain therapies have been little-studied in elderly populations. A variety of self-management programs aim to enhance the ability of patients to successfully self manage their pain, using a variety of techniques [72,73]. The most common behavioral modes of therapy include self-regulation strategies such as relaxation, biofeedback, hypnosis, imagery, and meditation. Although there are variations among these approaches, they share some or all of the following components: 1) education about pain and its consequences; 2) relaxation skills training (e.g., progressive muscle relaxation); 3) cognitive coping skills training; 4) problem solving (e.g., addressing problems with homework exercises or goals that are proposed to be met after each class); and 5) communication skills training (e.g., how to talk to physicians or health care providers about pain). In pain management, self management therapy serves to focus a patient's attention to exercise control in decreasing sympathetic arousal $[37,74]$. Besides patient education caregiver education is especially important for caring in the elderly. Both one-on-one as well as group programs can be effective $[3,7]$.

\subsubsection{Cognitive-behavioral therapy}

The American Psychological Association recognizes cognitive-behavioral therapy (CBT) as an empirically supported intervention in management of chronic musculoskeletal pain; including rheumatoid arthritis, osteoarthritis, fibromyalgia, and low back pain. Its foundation is the gate control theory integrating the sensory, affective, and cognitive components of pain. Cognitive processes are thoughts, self-statements, or evaluations about the pain and beliefs, interpretations, or attributions regarding this condition [37,75]. 10session psychosocial (i.e. cognitive behavioral orientation) pain management program that was specifically designed for older adults was used in ninety-five community dwelling seniors with at least one chronic musculoskeletal pain condition. Although decreases in pain 
intensity were observed in both the treatment and wait-list control groups, the intervention was found to result in fewer maladaptive beliefs about pain and greater use of relaxation, which is considered to be an adaptive coping strategy [76]. Beissner et al reported if physical therapists incorporate CBT techniques (eg, relaxation, activity pacing) when treating older patients with chronic pain. Commonly used CBT interventions included activity pacing and pleasurable activity scheduling [75].

\subsubsection{Mind-body therapies}

The National Center for Complementary and Alternative Medicine defines mind-body medicine in the following way: Mind-body medicine focuses on the interactions among the brain, mind, body, and behavior, and the powerful ways in which emotional, mental, social, spiritual, and behavioral factors can directly affect health. It regards as a fundamental approach that respects and enhances each person's capacity for self-knowledge and selfcare, and it emphasizes techniques that are grounded in this approach [72]. Morone et al conducted a structured review of eight mind-body interventions: biofeedback, progressive muscle relaxation, meditation, guided imagery, hypnosis, tai chi (TC), qi gong, and yoga for older adults with chronic nonmalignant pain. He reported that there is some support for the efficacy of progressive muscle relaxation plus guided imagery for osteoarthritis pain. There is limited support for meditation and TC for improving function or coping in older adults with low back pain or osteoarthritis. TC, yoga, hypnosis, and progressive muscle relaxation were significantly associated with pain reduction in these studies [72].

It is reported that prevalent coping strategies included analgesic medications (78\%), exercise (35\%), cognitive methods (37\%), religious activities (21\%), and activity restriction (20\%) for older adults with chronic pain due to a musculoskeletal cause [77]. Reid et al suggested in their review $(\mathrm{N}=27)$ that a broad range of self-management programs (yoga, massage therapy, TC, and music therapy) may provide benefits for older adults with CMP highlighting the need for research to establish the efficacy of the programs in different age and ethnic groups of older adults and identify strategies that maximize program reach longterm participation [74].

\subsubsection{Biologically based therapies}

Biologically based therapies, one of the major categories of complementary and alternative therapies, according to the federal National Institutes of Health (NIH), involve supplementing a person's normal diet with additional extracts, nutrients, herbs and/or certain foods. Among older adults, glucosamine sulfate and chondroitin sulfate are popular supplements used for the treatment of osteoarthritis and are among the most well studied biologic alternative medicines. Glucosamine and chondroitin are components of the extracellular matrix of articular cartilage; glucosamine is a substrate required to synthesize glycoproteins and glycosaminoglycans, components of synovial fluid, ligaments, and other cartilaginous joint structures, and chondroitin is a glycosaminoglycan that functions as a building block for joint matrix structure. Another commonly used biological agent for 
arthritis is S-adenosyl L-methionine (SAMe). This is a synthetic version of a naturally occurring coenzyme that is produced by the liver from methionine SAMe has been attributed with analgesic and antiinflammatory properties, and can stimulate articular growth [50].

\subsection{Exercise}

In recent years exercise, which is one of the non-pharmacological approaches, is getting the most important component of CMP management. Regular exercise, interventions to increase physical activity, strengthening the muscles, accompanied with weight loss are effective methods in the management of CMP such as OA, low back pain etc. in older adults. Regular moderate level exercise training or increased physical activity does not aggravate pain and joint symptoms as expected in OA according to RCTs and elicit significant health benefits. But pain, swelling, fatigue and weakness during activity or lasting more than 1-2 hours after exercise should be always considered as sign of excessive stress. Any activity that worsens pain or the other symptoms, and in acute flare-up periods of rheumatoid arthritis should be discontinued $[78,79]$.

The most studied CMP among older adults in literature belongs to knee OA. High and lowintensity aerobic exercises are equally effective in improving pain in persons with knee OA [80]. Specifically, aerobic exercise, water-based (aquatic) and land-based exercises, aerobic walking, quadriceps strengthening, and resistance exercise, physiotherapy-based exercise modalities reduce knee pain in older adults [80-84]. But a recent systematic review states that there are few RCTs recommending the use of exercise in reducing pain related to hip and knee $\mathrm{OA}$ and the content, duration and frequency of the exercise sessions is very heterogeneous [85].

\subsubsection{Benefits of exercise in chronic musculoskeletal pain}

Regular exercise also as an important adjunct to other interventions (e.g. thermal agents, patient education, etc.) is the most frequently preferred pain management strategies after medication in some older adult populations [55,58].

Various forms of exercise can modulate pain either directly or indirectly. Passive or active exercise has a direct effect on pain through increasing input from joint mechanoreceptors. Indirect effects of exercise on pain may be related to increased blood flow, decreased edema, inhibition of muscle spasm, enhanced ROM, flexibility, strength and weight loss which may improve biomechanical factors and decrease joint stress, and provide [58,81,86,87]. Improved sleep, enhanced mood, relaxation, reduction in anxiety and general well-being following regular exercise also can alter pain sensitivity positively in same way. After a single exercise session pain tolerance increases significantly $[58,88]$.

Another benefit of exercise is its effect on risk of falling among older adults with CMP. Older adults with CMP are at increased risk of falling because of pain related muscle weakness, increased body sway and impaired balance $[33,89,90]$. Primarily strengthening 
program and physical agents as an adjunct are recommended for joint pain management among this population [90]. The most effective physical therapy approach for the prevention of falls is a combination of balance and strength training [91] in addition to aerobic training such as walking, aerobic dance, circuit training, aquatics and active lifestyle [92]. RCTs are needed to learn whether pain reduction with exercise could affect fall risk in older adults with CMP.

\subsubsection{Types of exercise used in chronic musculoskeletal pain}

An exercise program should address primer functional problems and impairments (pain, limited joint range of motion, muscle weakness) for functional independence. After relieving from these impairments or reducing them exercise program can begin [78]. A physical therapist has the primary responsibility to plan an exercise program accommodating pain or other disabilities [93]. Flexibility, strength and aerobic endurance are the basic components for exercise programs aiming to control pain. Time needed for adaptation to exercise stress may be 2 to 3 months for older arthritic adults with low physical capacity [78].

Exercise sessions should have three phases: The first phase, a warm-up period lasting 5-10 minutes, involves repetitive low-intensity range-of-motion exercises. The second phase, the training period, includes range of motion, strength, or aerobic capacity exercises, or a combination of these. The final phase, cool-down period lasting 5 minutes, involves flexibility exercises $[78,94]$. In addition the time of the exercise during the day can change according to the chronic condition. Older adults with OA better perform exercise in the morning, whereas older adults with rheumatoid arthritis may be better several hours after awakening. Low-impact, non-weight-bearing exercises and exercise machines distributing the load to all limbs usually recommended for artritic patients [79].

\subsubsection{Flexibility exercises}

Flexibility exercise should begin at the beginning of an exercise program during the warmup, preferably cool-down period. Static stretching is recommended during cool down period for the osteoartritic older adults at as full as possible pain-free range for the greatest improvements [95]. Stretching exercises must be modified when the joint is inflamed or painful. Painful joints should not be over stretched and superficial heat application, relaxation prior to stretching helps reduce pain [78]. Older adults tend to have some movement patterns and positioning, which causes joint movement limitation resulting in painful movement patterns. Consideration of the potential for future painful conditions also should be treated by stretching [96].

Stretching exercises should be performed at least 3 times per week or daily if the pain and stiffness are minimal or must be modified when the joint is inflamed or painful. The progression should be gradual from one stretching to 4-10 repetitions for each major muscle group. The stretch position should be hold 10-30 seconds. [78]. Effective stretching exercises require longer holding times with increasing age and loss of extensibility, so if there is no pain, 
60 seconds is necessary for older adults to achieve a long-term effect. Four repetitions of a 60second hold performed regularly, 5 to 7 days a week, appear to be most effective [96].

\subsubsection{Aerobic exercises}

Aerobic exercise programs aiming improvement in strength and proprioception reduce pain in OA patients. Examples of aerobic exercise are bicycling (stationary bike, recumbent-type bike etc.), walking, dance, Tai-Chi and aquatic exercises such as swimming, Ai-Chi etc. Daily activities and some hobbies like walking the dog, mowing the lawn or playing golf, are also considered as aerobic exercise [78,92]. To prevent overuse of specific joints and to elicit long-term participation, activity selection for aerobic exercise is important and depends on the patient's current disease state, joint stability, opportunities, individual's preference and abilities [96].

There are few studies addressing effect of aerobic exercise on CMP in older adults [97, 98]. A 14-year prospective longitudinal study showed that regular aerobic exercise over the long period in physically active seniors was associated with about $25 \%$ less CMP than reported by more sedentary ones [97].

The aerobic exercise intensity should range between 50\%-60\% of HRmax (220 - age in years), 10-12 point in rating of perceived exertion (an ordinal scale, 6 to 20), or be positive on the "talk test" [78]. The talk test represents the ability to engage in a conversation during exercise. When the exerciser reaches an intensity at which he or she can "just barely respond in conversation," the intensity is considered to be safe and appropriate for cardiovascular adaptation [96]. The initial intensity may be 9-11 point in rating of perceived exertion for frail and sedentary older adults [92]. A $2.5 \%$ increase in the intensity or volume weekly is appropriate for adaptation and prevention of musculoskeletal injuries among arthritic older adults [78].

The ideal volume for the beginner is 20 to 30 minutes per day but for sedentary, frailer or more deconditioned older adults, it would be easier to begin with one to five exercise bouts of 3-5 minutes in a day and gradually reach to ideal length. Totally 60 and 90 minutes of moderate level physical activity during a week is recommended by the ACSM (American Colleges of Sports Medicine) [78,92].

The initial frequency of exercise training is recommended $3[78,92]$ and later maximum 4 days a week in order not to cause injury according to ACSM [78].

\subsubsection{Strengthening exercise}

Joint pain can limit older adults from contracting multiple muscles to provide a cardiovascular stimulus during aerobic exercise and causes muscle weakness. In those cases and for frail older adults, it is sensible to add aerobic activity following strengthening and balance exercise to stabilize or support the joint and decrease pain followed by functional improvement $[15,82,83,92,96]$. Both high and low intensity resistive training significantly 
reduces pain [99]. A Cochrane systematic review showed that there was evidence for modest reduction in pain following progressive resistive training. It is also reported that there was no significant difference in reducing pain between progressive resistive training with functional, aerobic and flexibility training [100,101].

Because low articular pressures during isometric contractions can be well tolerated, isometric strengthening with a few repetition should be given if the joints are inflamed, unstable, swollen, painful or if it is initial phase of strengthening program [78,79]. Isometric strength training should target the major muscle groups. The intensity should gradually increase to $75 \%$ from approximately $30 \%$ of the maximal voluntary contraction; the number of repetitions to 8-10 from one; number of sessions to 5-10 from 2 times throughout the day. During a contraction held for maximum 6 seconds $(20$ seconds resting between contractions), older adults should keep on breathing. Contractions should be performed at different muscle lengths or joint angles, too [78]. As soon as possible, when it is tolerated, isotonic training involving 8-10 major muscle groups should begin to improve overall function maximum of 2 days a week. The intensity should gradually increase to $80 \%$ from $40 \%$ of $1 \mathrm{RM}$ (repetition maximum) for adaptation [78]. 1RM is the weight a person can lift one time with good form. The ACSM recommends no more than three trials with a 30- to 60second rest between trials to find out the most accurate $1 \mathrm{RM}$, but older adults may have a better response with a multiple RM of 6 to 10 because they need experience to learn to generate that type of force. Elastic bands or tubing, cuff and hand weights, barbells, dumbbells, hand-held blades, fixed weights, medicine and stability balls etc. can be used as equipment $[96,102]$.

For safety reasons, older adults especially those with cardiovascular problems adults should not perform more than two to three sets of a given exercise and repetition number must be carefully determined. For muscular endurance sets of 12-15 repetitions with lighter resistances, for strength development 8-12 repetitions with higher resistance should be used [78].

Another option for exercise is stabilization exercises, which target co-activation of specific muscles and provide joint stability based on the spine [103]. Increased strength and crosssectional area of the vertebral muscles reduces CLBP by maintaining muscle balance [102].

\subsubsection{Tai-Chi}

Tai-Chi [TC], shortly defined as a traditional Chinese mind-body exercise, has recently become popular worldwide because more people with musculoskeletal problems are looking for complementary and alternative treatments [104,105]. TC gives emphasis to diaphragmatic breath, relaxation and composed of slow, gentle, smooth, harmonic and coordinated movements of different body parts, and weight shifting [104,106]. TC involves routines or "forms" ranging from the classic 109 postures to as few as 42 and now has multiple styles modified from the original form. In addition to physical benefits, the focus required to complete these routines elicits mental and cognitive benefits [96]. 
TC is a moderate-intensity exercise, so it is suitable for physically frail older adults. Besides reducing the pain, people practice TC for also improving physical condition, muscle strength, coordination, flexibility, balance, decreasing risk for falls, stiffness, fatigue, improving sleep, cardiovascular and respiratory function, mood, depression, anxiety, selfefficacy, health-related quality of life and overall wellness in both eastern and western populations $[103,104,106,107]$. The therapeutic benefits of TC for chronic conditions have been showed in researches recently.

TC is common in older adults especially those with OA, because it is shown to improve pain $[103,108]$, although it is stated that the methodological quality of TC research is generally less than strength and aerobic training research [84]. The physical component of TC provides current recommendations for OA (strength, balance, flexibility, and aerobic cardiovascular exercise) and the mental component could contribute to chronic pain reduction by modulating complex factors of OA pain [104].

Significant pain-relieving effect is shown especially at knee rather than other joints like in the upper extremities where less weight-carrying activity involved in TC [109-111]. It is believed that weight-carrying TC footwork provides pain-relieving effects on knee OA [106]. TC also showed no significant difference in pain reduction of older adults with knee and hip OA compared to hydrotherapy, where there is less knee joint stress than TC [112].

A systematic review and meta-analysis suggested that TC had a small positive effect on pain in people with arthritis and the extent to which it benefits other forms of CMP is unclear but the review also reported that the studies included were low-quality [108]. However a more recent study indicates that water- or land-based exercise, aerobic walking, quadriceps strengthening, resistance exercise, and TC reduce pain and disability from knee OA with evidence rating of A category [80].

There is another discussion about TC that if its benefit increases when combined with the other exercise types or not. Yip YB et al showed that self-management exercise program including stretching, walking, and TC types of movement, had positive effect in reducing pain [113] but a recent systematic review concluded that TC based exercise programs elicited better outcomes than mixed ones but without clear differences [85].

Among different TC styles (Chen, Yang, Wu styles...ect.) the "Sun" style is the most studied one. Sun style TC requires higher stance with bending knees less than other types, so it is more comfortable. In fact in all styles the patient can prefer high or low stance [106]. Song $R$ et al reported that a Sun-style TC exercise could be applied to OA patients in outpatient clinics or public health centers if they are not in acute inflammatory stage to reduce arthritic symptoms [114]. Simplified Yang-style TC is also shown to be effective in osteoartritic knee pain $[115,116]$.

Beside osteoartritis, benefits of TC have been found in some other musculoskeletal problems such as fibromyalgia [6], rheumatoid arthritis [117] and nonspecific CLBP [118]. 


\subsubsection{Aquatic exercise}

Aquatic exercise is another good option for the treatment of musculoskeketal problems because water is a safe exercise environment and its temperature provides analgesia for painful muscles and joints $[78,119]$. The water temperature is recommended between 85 and 90 Fahrenheit (29-32 Celsius) for artritic older adults [79].

The buoyancy of water causes less impact or compressive forces on the joints and therefore allows pain-free motion without the biomechanical stress experienced on land [119]. Older adults with OA or history of surgery may benefit from aquatic exercise. Water resistance can be used for strengthening to progress to land-based exercise among older adults with arthritis. Moreover aquatic exercise, usually practiced with a group, motivates practitioners $[78,96]$. It is reported that among older adults class attendance is higher for hydrotherapy compared with TC [112] because it provides a playful environment, many social and psychological benefits for them [119]. It also should be considered that heart rate is lower than heart rates when performing at the same level of oxygen consumption on land. "Aquatic heart rate reduction" should be included in the formula while determining target heart rate or it is sensible to use rating of perceived exertion when determining aquatic exercise intensity $[96,119]$.

\subsubsection{Exercise adherence in older adults with chronic musculoskeletal pain}

Chronic pain has been found to be associated with difficulty in exercising regularly [120]. Motivation has a key role for older adults to participate in exercise willingly. Older adults' outcome and self-efficacy expectations, negative sensations associated with physical activity, such as fear of pain especially back pain or falling influence motivation to engage in physical activities [121]. These negative sensations and related beliefs must be eliminated through facilitating appropriate use of pain medications before exercise or alternative measures such as heat/ice before or after exercise to relieve activity related pain, use of braces or straps, or isolating the damaged joint during exercise. Additionally positive reinforcement and self-management interventions including explaining to older adults how exercise will help reduce pain, cognitive-behavioral therapy, relaxation and distraction techniques and graded exposure to overcome fear of falling or pain can improve participation to exercise among older adults with arthritic pain. Even pain should be minimized in every way possible, the older adult may have to learn to tolerate some pain or discomfort $[73,79,86,121]$.

The use of supervised exercise sessions such as classes in the initial exercise period followed by home exercises and calling patients back for intermittent consultations, or "refresher" group exercise classes may also assist long-term adherence [83]. Generally older adults are interested in self-managing their chronic pain but can't find opportunity. Austrian et al indicated that $73 \%$ of the 68 patients (70 years of age and over with chronic pain) included in his study were willing to participate in an exercise program for pain management but $16 \%$ of them had this opportunity [40]. 
Most types of exercise with some evidence are frequently preferred for pain management in older adult populations with CMP especially for arthritis, mostly knee OA, and secondly CLBP. Exercise content, time and frequency are very heterogeneous in RCTs, so it is hard to determine the best exercise structure. At that point individualized approach to exercise prescription is required.

\section{Conclusion}

Because aging is an extremely variable process, older adults require more individualized management than younger individuals. Treatment decisions should weigh the risks of pain with the risks of treatment. In order to provide the most efficient and safest therapy approach in the older adults with musculoskeletal pain, the identification and frequent reevaluation of the cause of the chronic pain and the impact on the patient's general medical state are crucial.

The high cost and adverse side effect profiles associated with many analgesic treatments, as well as the potential for drug-drug interactions, operate as significant barriers to the use of standard pharmacologic treatments in older adults $[19,74]$. Based on studies conducted to date, combined pharmacologic and non- pharmacologic therapies give the best results for pain relief. Regardless, alternative or complementary medical interventions should be recognized as options for older adults with chronic musculoskeletal pain $[4,56]$. While some studies have demonstrated that integrating complementary medicine into the care of older patients can yield promising results. Additionally, some of the challenges encountered with conventional pain management of older adults can be ameliorated by integrating complementary and alternative medicine approaches [56].

\section{Author details}

Ayse Ozcan Edeer

Adjunct Faculty, Doctoral Program in Physical Therapy, Dominican College, NY, USA

Hulya Tuna

School of Health, Department of Physiotherapy and Rehabilitation, Izmir University, Izmir, Turkey

\section{References}

[1] Ferrell BA, (2001) Pain management in the elderly. Clin Geriatr Med. 17:417-615.

[2] Keefe FJ, Beaupre PM, Weiner DK, Siegler IC (1996) Pain in older adults: a cognitivebehavioral perspective. In: Ferrell BR, Ferrell BA. Pain in the Elderly. Wash: IASP Press. Pp:11-19.

[3] Cavalieri TA (1999) Pain management at the end of life. J Am Osteopath Assoc. 99(6): 16-21.

[4] Gloth MJ and Black RA (2011) The Role of Rehabilitation in Managing Pain in Seniors. In Gloth FM (2011) Handbook of pain relief in older adults: an evidence-based 
approach (1-60761-617-3, 978-1-60761-617-7), 2nd ed. DOI 10.1007/978-1-60761-618-4. P:45

[5] Schofield P, Black C, Aveyard B (2011). Management of Pain in Older People. Humana Press.

[6] Brown ST, Kirkpatrick MK, Swanson MS, McKenzie IL (2011) Pain experience of the elderly. Pain Manag Nurs. 12(4):190-6.

[7] Cavalieri TA (2002) Pain management in the elderly. JAOA. 102(9):481-485

[8] http://www.dh.gov.uk/en/Publicationsandstatistics/Publications/PublicationsPolicyAnd Guidance/DH_4010161

[9] D'Astolfo CJ and Humphreys BK (2006) A record review of reported musculoskeletal pain in an Ontario long term care facility. BMC Geriatrics. 6:5. doi:10.1186/1471-2318-6-5.

[10] Williams AK (1999) Geriatric Rehabilitation Manual, In: Kaufman TL editor. Pain. Churchill Livinstone. pp:359-362.

[11] Cowan DT, Fitzpatricka JM, Robertsa JD, Whileb AE, Baldwin J et al. (2003) The assessment and management of pain among older people in care homes: current status and future directions. International Journal of Nursing Studies. 40 291-298.

[12] Arnstein P (2010) Balancing analgesic efficacy with safety concerns in the older patient. Pain Manag Nurs. 11(2 Suppl):11-22.

[13] Gloth F (2001) Pain Management in Older Adults: Prevention and Treatment. J Am Geriatr Soc. 49:188-199.

[14] http://www.iasp-pain.org,

[15] Tse MM, Wan VT, Ho SS (2011) Physical exercise: does it help in relieving pain and increasing mobility among older adults with chronic pain? J Clin Nurs. 20(5-6):635-44.

[16] AGS Panel on Persistent Pain in Older Persons. The management of persistent pain in older persons. (2002) J Am Geriatr Soc. 50(6 Suppl):205-224.

[17] Herr KA, Garand L (2001) Assessment and measurement of pain in older adults. Clin Geriatr Med. 17:457-478.

[18] Herr K (2010) Pain in the older adult: an imperative across all health care settings. Pain Manag Nurs. 11(2 Suppl):1-10.

[19] Ersek M, Turner JA, Cain KC and Kemp CA (2004) Chronic pain self-management for older adults: a randomized controlled trial. BMC Geriatrics 4:7. doi:10.1186/1471-2318-4-7

[20] Beissner K (2012) Conservative Pain Management for the Older Adult. In: Guccione A, Wong R, Avers D, editors. Geriatric Physical Therapy. 3rd ed. Elsevier. Pp:395-411.

[21] Charlton JE (2005) Pain in Older Adults Core Curriculum for Professional Education in Pain, IASP Press, (4):1-4.

[22] Frondini C, Lanfranchi G, Minardi M, Cucinotta D (2007) Affective, behavior and cognitive disorders in the elderly with chronic musculoskelatal pain: the impact on an aging population. Arch Gerontol Geriatr. 44 (Suppl 1):167-71.

[23] Podichetty VK, Mazanec DJ, Biscup RS (2003) Chronic non-malignant musculoskeletal pain in older adults: clinical issues and opioid intervention. Postgrad Med J. 79(937):627-33.

[24] Yamada E, Thomas DC (2011) Common musculoskeletal diagnoses of upper and lower extremities in older patients. Mt Sinai J Med. 78(4):546-57. doi: 10.1002/msj.20274. 
[25] Morone NE, Karp JF, Lynch CS, Bost JE, El Khoudary SR, Weiner DK (2009) Impact of chronic musculoskeletal pathology on older adults: a study of differences between knee OA and low back pain. Pain Med. 10(4):693-701.

[26] Weiner DK, Cayea D (2005) Low back pain and its contributors in older adults: a practical approach to evaluation and treatment. In: Gibson SJ, Weiner DK, eds. Pain in Older Persons, Progress in Pain Research and Management. IASP Press. (35)329-354.

[27] Rudy TE, Weiner DK, Lieber SJ, Slaboda J, Boston JR (2007) The impact of chronic low back pain on older adults: a comparative study of patients and controls. Pain. 131(3):293-301.

[28] Leveille SG, Zhang Y, McMullen W, Kelly-Hayes M, and Felson DT (2005) Sex differences in musculoskeletal pain in older adults. Pain. 116(3): 332-338.

[29] Shah RC, Buchman AS, Boyle PA, Leurgans SE, Wilson RS, Andersson GB, Bennett DA (2011) Musculoskeletal pain is associated with incident mobility disability in community-dwelling elders. J Gerontol A Biol Sci Med Sci. 66(1):82-8.

[30] Buchman AS, Shah RC, Leurgans SE, Boyle PA, Wilson RS, Bennett DA. (2010) Musculoskeletal pain and incident disability in community-dwelling older adults. Arthritis Care Res. 62(9):1287-93.

[31] Mitchell C (2001) Assessment and management of chronic pain in elderly people. Br J Nurs. 8-21;10(5):296-304.

[32] Leveille SG, Bean J, Bandeen-Roche K, Jones R, Hochberg M, Guralnik JM (2002) Musculoskeletal pain and risk for falls in older disabled women living in the community. J Am Geriatr Soc. 50(4):671-8.

[33] Leveille SG, Jones RN, Kiely DK, Hausdorff JM, Shmerling RH, Guralnik JM, Kiel DP, Lipsitz LA, Bean JF (2009) Chronic musculoskeletal pain and the occurrence of falls in an older population. JAMA. 25;302(20):2214-21.

[34] Mesrine S, Boutron-Ruault MC, Clavel-Chapelon F (2010) Chronic pain and risk of falls in older adults. JAMA. 24;303(12):1147-8.

[35] Wilber ST, Sullivan AF, Camargo CA Jr (2010) Chronic pain and risk of falls in older adults. JAMA. 24:303(12):1148-9.

[36] Lihavainen K, Sipilä S, Rantanen T, Sihvonen S, Sulkava R, Hartikainen S (2010) Contribution of musculoskeletal pain to postural balance in community-dwelling people aged 75 years and older. J Gerontol A Biol Sci Med Sci. 65(9):990-6.

[37] Golden BA (2002) A multidisciplinary approach to nonpharmacologic pain management. JAOA. Supplement 3 102(9):1-5.

[38] Cayea D, Perera S, Weiner DK (2006) Chronic low back pain in older adults: What physicians know, what they think they know, and what they should be taught. J Am Geriatr Soc. 54(11):1772-7.

[39] Leveille SG, Bean J, Ngo L, McMullen W, Guralnik JM (2007) The pathway from musculoskeletal pain to mobility difficulty in older disabled women. Pain. 128(1-2):69-77.

[40] Austrian JS, Kerns RD, Reid MC (2005) Perceived barriers to trying self-management approaches for chronic pain in older persons. J Am Geriatr Soc. 53(5):856-61.

[41] Bruckenthal P, Reid C, and Reisner L (2009) Special Issues in the Management of Chronic Pain in Older Adults. Pain Medicine. 10:67-78. 
[42] Ballentine NH (2008) Polypharmacy in the elderly: maximizing benefit, minimizing harm. Crit Care Nurs Q. 31(1):40-5.

[43] McPherson ML and Uritsky TJ (2011) Pharmacotherapy of Pain in Older Adults: Nonopioid. In Gloth FM Handbook of pain relief in older adults: an evidence-based approach (1-60761617-3, 978-1-60761-617-7), 2nd ed. DOI 10.1007/978-1-60761-618-4. P:57

[44] Saad M, Harisingani R, Katinas L (2012) Impact of geriatric consultation on the number of medications in hospitalized older patients. Consult Pharm. 27(1):42-8.

[45] Elliott AF, Horgas AL (2009) Effects of an analgesic trial in reducing pain behaviors in community-dwelling older adults with dementia. Nurs Res. 58(2):140-5.

[46] Kean WF, Rainsford KD, Kean IR (2008) Management of chronic musculoskeletal pain in the elderly: opinions on oral medication use. Inflammopharmacology. 16(2):53-75.

[47] Cooper JW, Burfield AH (2003) Assessment and management of chronic pain in the older adult. J Am Pharm Assoc. 2010 50(3):89-99.

[48] Muller S, Bedson J, Mallen CD (2012) The association between pain intensity and the prescription of analgesics and non-steroidal anti-inflammatory drugs. Eur J Pain. 19. doi: 10.1002/j.1532-2149.2011.00107.x.

[49] Taylor R Jr, Lemtouni S, Weiss K, Pergolizzi JV Jr (2012) Pain Management in the Elderly An FDA Safe Use Initiative Expert Panel's View on Preventable Harm Associated with NSAID Therapy. Curr Gerontol Geriatr Res. 2012:196159.

[50] Bruckenthal P (2010) Integrating Nonpharmacologic and Alternative Strategies Into a Comprehensive Management Approach for Older Adults With Pain. Pain Management Nursing. 11(2):23-31

[51] Trescot AM, Helm S, Hansen H, Benyamin R, Glaser SE, Adlaka R, Patel S, Manchikanti $\mathrm{L}$ (2008) Opioids in the management of chronic non-cancer pain: an update of American Society of the Interventional Pain Physicians' (ASIPP) Guidelines. Pain Physician. 11(2 Suppl):5-62.

[52] Hicks GE, Shardell M, Miller RR, Bandinelli S, Guralnik J, Cherubini A, Lauretani F, Ferrucci L (2008) Associations between vitamin D status and pain in older adults: the Invecchiare in Chianti study. J Am Geriatr Soc. 56(5):785-91.

[53] Fialová D, Onder G (2009) Medication errors in elderly people: contributing factors and future perspectives. Br J Clin Pharmacol. 67(6):641-5.

[54] Planton J, Edlund BJ (2010) Strategies for reducing polypharmacy in older adults. J Gerontol Nurs. 36(1):8-12. doi: 10.3928/00989134-20091204-03.

[55] Kemp CA, Ersek M, Turner JA (2005) A descriptive study of older adults with persistent pain: use and perceived effectiveness of pain management strategies. BMC Geriatr. 8:5:12 doi:10.1186/1471-2318-5-12.

[56] Bruckenthal P (2010) Integrating nonpharmacologic and alternative strategies into a comprehensive management approach for older adults with pain. Pain Manag Nurs. 11(2 Suppl):23-31.

[57] Komp-Webb M (2008) Physical Therapy. In ed MP Jansen. Managing pain in the older adult. Ed Springer Pub. Co. P:93-117

[58] Barr JO (2007) Conservative interventions for pain control. In: Kauffman TL, Barr JO, Moran ML (eds). Geriatric Rehabilitation Manual (2nd ed), Churchill Livingstone.P:449455 
[59] Barry LC, Gill TM, Kerns RD, Reid MC (2005) Identification of pain-reduction strategies used by community-dwelling older persons. J Gerontol A Biol Sci Med Sci. 60(12):1569-75.

[60] Chatap G, De Sousa A, Giraud K, Vincent JP (2007) Acute Pain in the Elderly Study Group. Pain in the elderly: Prospective study of hyperbaric CO2 cryotherapy (neurocryostimulation). Joint Bone Spine. 74(6):617-21.

[61] French HP, Brennan A, White B, Cusack T (2011) Manual therapy for osteoarthritis of the hip or knee - A systematic review. Manual Therapy. 16(2):109-117.

[62] Miller J, Gross A, D'Sylva J, Burnie SJ, Goldsmith CH, Graham N, Haines T, Brønfort G, Hoving JL (2010) Manual therapy and exercise for neck pain: A systematic review. Manual Therapy.15:33-35.

[63] Harris M, Richards KC (2010) The physiological and psychological effects of slowstroke back massage and hand massage on relaxation in older people. J Clin Nurs. 19(78):917-26.

[64] Hawk C, Rupert RL, Colonvega M, Boyd J, Hall S (2006) Comparison of bioenergetic synchronization technique and customary chiropractic care for older adults with chronic musculoskeletal pain. J Manipulative Physiol Ther. 29(7):540-9.

[65] van Middelkoop M, Rubinstein SM, Kuijpers T, Verhagen AP, Ostelo R, Koes BW, van Tulder MW (2011) A systematic review on the effectiveness of physical and rehabilitation interventions for chronic non-specific low back pain. Eur Spine J. 20(1):19-39.

[66] Vieira PJ, Ribeiro JP, Cipriano G Jr, Umpierre D, Cahalin LP, Moraes RS, Chiappa GR (2012) Effect of transcutaneous electrical nerve stimulation on muscle metaboreflex in healthy young and older subjects. Eur J Appl Physiol. 112(4):1327-34.

[67] Weiner DK, Rudy TE, Glick RM, Boston JR, Lieber SJ, Morrow LA, Taylor S (2003) Efficacy of percutaneous electrical nerve stimulation for the treatment of chronic low back pain in older adults. J Am Geriatr Soc. 51:599-608.

[68] Zhang W, Moskowitz RW, Nuki G, Abramson S, Altman RD, Arden N, Bierma-Zeinstra S, Brandt KD, Croft P, Doherty M, Dougados M, Hochberg M, Hunter DJ, Kwoh K, Lohmander LS, Tugwell P (2008) OARSI recommendations for the management of hip and knee osteoarthritis, Part II: OARSI evidence-based, expert consensus guidelines. Osteoarthritis Cartilage. 16(2):137-62.

[69] Zhang W, Nuki G, Moskowitz RW, Abramson S, Altman RD, Arden NK, BiermaZeinstra S, Brandt KD, Croft P, Doherty M, Dougados M, Hochberg M, Hunter DJ, Kwoh K, Lohmander LS, Tugwell P (2010) OARSI recommendations for the management of hip and knee osteoarthritis: part III: Changes in evidence following systematic cumulative update of research published through January 2009. Osteoarthritis Cartilage. 18(4):476-99.

[70] Barnes PM, Bloom B, Nahin RL (2008) Complementary and Alternative Medicine Use Among Adults and Children: United States, 2007, National Health Statistics Reports Number 12, December 10.

[71] Molton I, Jensen MP, Ehde DM, Carter GT, Kraft G, Cardenas DD (2008) Coping with chronic pain among younger, middle-aged, and older adults living with neurological injury and disease. J Aging Health. 20(8):972-996. doi:10.1177/0898264308324680.

[72] Morone NE, and Greco CM (2007) Mind-Body Interventions for Chronic Pain in Older Adults:A Structured Review. Pain Medicine. 8(4):359-75. 
[73] Nour K, Laforest S, Gauvin L, Gignac M (2006) Behavior change following a selfmanagement intervention for housebound older adults with arthritis: an experimental study. It J Behav Nutr Phys Act. 30:3:12.

[74] Reid MC, Papaleontiou M, Ong A, Breckman R, Wethington E, Pillemer K (2008) Selfmanagement strategies to reduce pain and improve function among older adults in community settings: a review of the evidence. Pain Med. 9(4):409-24.

[75] Beissner K, Henderson CR Jr, Papaleontiou M, Olkhovskaya Y, Wigglesworth J, Reid MC (2009) Physical therapists' use of cognitive-behavioral therapy for older adults with chronic pain: a nationwide survey. Phys Ther. 89:456-469.

[76] Green SM, Hadjistavropoulos T, Hadjistavropoulos H, Martin R, Sharpe D (2009) A Controlled Investigation of a Cognitive Behavioral Pain Management Program for Older Adults Behavioral and Cognitive Psychotherapy. 37:221-226.

[77] Barry LC, Kerns RD, Guo Z, Duong BD, Iannone LP, Reid MC (2004) Identification of strategies used to cope with chronic pain in older persons receiving primary care from a Veterans Affairs Medical Center. J Am Geriatr Soc. 52(6):950-6.

[78] American Geriatrics Society Panel on Exercise and Osteoarthritis. Exercise prescription for older adults with osteoarthritis pain: consensus practice recommendations (2001) A supplement to the AGS Clinical Practice Guidelines on the management of chronic pain in older adults. J Am Geriatr Soc. 49(6):808-823.

[79] Rimmer JH (2005) Exercise Considerations for Medical Conditions. In: Jones CJ, Rose DJ, editors. Physical Activity Instruction of Older Adults. Human Kinetics. pp. 336-349.

[80] Ringdahl E, Pandit S (2011) Treatment of knee osteoarthritis. Am Fam Physician. 83(11):1287-1292.

[81] Bosomworth NJ (2009) Exercise and knee osteoarthritis: benefit or hazard? Can Fam Physician. 55(9):871-878.

[82] Ettinger WH, Burns R, Messier SP, Applegate W, Rejeski WJ, Morgan T, Shumaker S, Berry MJ, O'Toole M, Monu J, Craven T (1997) A randomized trial comparing aerobic exercise and resistance exercise with a health education program in older adults with knee osteoarthritis. The Fitness Arthritis and Seniors Trial (FAST). JAMA. 277(1):25-31.

[83] Roddy E, Zhang W, Doherty M (2005) Aerobic walking or strengthening exercise for osteoarthritis of the knee? A systematic review. Ann Rheum Dis. 64(4):544-548.

[84] Bennell KL, Hinman RS (2011) A review of the clinical evidence for exercise in osteoarthritis of the hip and knee. J Sci Med Sport.14(1):4-9.

[85] Escalante Y, Saavedra JM, García-Hermoso A, Silva AJ, Barbosa TM (2010) Physical exercise and reduction of pain in adults with lower limb osteoarthritis: a systematic review. J Back Musculoskelet Rehabil. 23(4):175-186.

[86] Resnick B (2001) Managing arthritis with exercise. Geriatr Nurs. 22(3):143-150.

[87] Messier SP, Loeser RF, Miller GD, Morgan TM, Rejeski WJ, Sevick MA, Ettinger WH Jr, Pahor M, Williamson JD (2004) Exercise and dietary weight loss in overweight and obese older adults with knee osteoarthritis: the Arthritis, Diet, and Activity Promotion Trial. Arthritis Rheum. 50(5):1501-1510.

[88] Bartholomew JB, Lewis BP, Linder DE, Cook DB (1996) Post-exercise analgesia: replication and extension. J Sports Sci.4(4):329-334. 
[89] Downton JH. Why do old people fall? Falls in the elderly. Great Britain: Hodder and Stoughton Limited; 1993: 1-77.

[90] Alghwiri AA, Whitney SL (2012) Balance and Falls. In: Guccione A, Wong R, Avers D, editors. Geriatric Physical Therapy. 3rd ed. Elsevier. pp. 331-353.

[91] Karinkanta S, Piirtola M, Sievänen H, Uusi-Rasi K, Kannus P (2010) Physical therapy approaches to reduce fall and fracture risk among older adults. Nat Rev Endocrinol. 6(7):396-407.

[92] Dinan S, Skelton D, Malbut K (2005) Aerobic Endurance Training. In: Jones CJ, Rose DJ, editors. Physical Activity Instruction of Older Adults. Human Kinetics. pp. 191-210.

[93] Brown M, Avers D, Wong RA (2012) Wellness for the Aging Adult-Special Populations and the Continuum of Care. In: Guccione A, Wong R, Avers D, editors. Geriatric Physical Therapy. 3rd ed. Elsevier. pp. 446-456.

[94] Norman KV (2005) Principles of The Warm-Up and Cool-Down. In: Jones CJ, Rose DJ, editors. Physical Activity Instruction of Older Adults. Human Kinetics. pp. 141-153.

[95] Brown M, Rose DJ (2005) Flexibility Training. In: J ones CJ, Rose DJ, editors. Physical Activity Instruction of Older Adults. Human Kinetics. pp. 156-174.

[96] VanBeveren PJ, Avers D (2012) Exercise and Physical Activity for Older Adults. In: Guccione A, Wong R, Avers D, editors. Geriatric Physical Therapy. 3rd ed. Elsevier. pp. 64-85.

[97] Bruce B, Fries JF, Lubeck DP (2005) Aerobic exercise and its impact on musculoskeletal pain in older adults: a 14 year prospective, longitudinal study. Arthritis Res Ther.7(6):1263-1270.

[98] Fries JF, Singh G, Morfeld D, O'Driscoll P, Hubert H (1996) Relationship of running to musculoskeletal pain with age. A six-year longitudinal study. Arthritis Rheum. 39(1):6472.

[99] Jan MH, Lin JJ, Liau JJ, Lin YF, Lin DH (2008) Investigation of clinical of high and low resiztance training for patients with knee osteoarthritis: a randomized controlled trial. Phys Ther. 88(4):427-436.

[100] Barrett CJ, Smerdely P (2002) A comparison of community-based resistance exercise and flexibility exercise for seniors. Aust J Physiother. 48(3):215-219.

[101] Liu CJ, Latham NK (2009) Progressive resistance strength training for improving physical function in older adults. Cochrane Database Syst Rev. 8(3):CD002759.

[102] Kraemer WJ, French DN (2005) Resistance Training. In: Jones CJ, Rose DJ, editors. Physical Activity Instruction of Older Adults. Human Kinetics. pp. 176-189.

[103] Christiansen C (2012) Impaired Joint Mobility. In: Guccione A, Wong R, Avers D, editors. Geriatric Physical Therapy. 3rd ed. Elsevier. Pp: 248-262.

[104] Wang C (2011) Tai Chi and Rheumatic Diseases. Rheum Dis Clin North Am. 37(1): 1932.

[105] Hawker GA, Mian S, Bednis K, Stanaitis (2011) I. Osteoarthritis year 2010 in review: non-pharmacologic therapy. Osteoarthritis Cartilage. 19(4):366-374.

[106] Chyu MC, von Bergen V, Brismée JM, Zhang Y, Yeh JK, Shen CL (2011). Complementary and alternative exercises for management of osteoarthritis. Arthritis. 2011:364319. 
[107] Ho TJ, Liang WM, Lien CH, Ma TC, Kuo HW, Chu BC, Chang HW, Lai JS, Lin JG (2007) Health-related quality of life in the elderly practicing T'ai Chi Chuan. J Altern Complement Med. 13(10):1077-1083.

[108] Hall A, Maher C, Latimer J, Ferreira M (2009) The effectiveness of Tai Chi for musculoskeletal pain conditions: a systematic review and meta-analysis. Arthritis Rheum. 61(6):717-724.

[109] Song R, Lee EO, Lam P, Bae SC (2003) Effects of tai chi exercise on pain, balance, muscle strength, and perceived difficulties in physical functioning in older women with osteoarthritis: a randomized clinical trial. J Rheumatol. 30(9):2039-2044.

[110] Wang C, Schmid CH, Hibberd PL, Kalish R, Roubenoff R, Rones R, McAlindon T (2009) Tai Chi is effective in treating knee osteoarthritis: a randomized controlled trial. Arthritis Rheum. 61(11):1545-1553.

[111] Brismée JM, Paige RL, Chyu MC, Boatright JD, Hagar JM, McCaleb JA, Quintela MM, Feng D, Xu KT, Shen CL (2007) Group and home-based tai chi in elderly subjects with knee osteoarthritis: a randomized controlled trial. Clin Rehabil. 21(2):99-111.

[112] Fransen M, Nairn L, Winstanley J, Lam P, Edmonds J (2007) Physical activity for osteoarthritis management: a randomized controlled clinical trial evaluating hydrotherapy or Tai Chi classes. Arthritis Rheum. 57(3):407-414.

[113] Yip YB, Sit JW, Fung KK, Wong DY, Chong SY, Chung LH, Ng TP (2007) Effects of a self-management arthritis programme with an added exercise component for osteoarthritic knee: randomized controlled trial. J Adv Nurs. 59(1):20-8.

[114] Song R, Lee EO, Lam P, Bae SC (2007) Effects of a Sun-style Tai Chi exercise on arthritic symptoms, motivation and the performance of health behaviors in women with osteoarthritis. Taehan Kanho Hakhoe Chi. 37(2):249-56.

[115] Ni GX, Song L, Yu B, Huang CH, Lin JH (2010) Tai chi improves physical function in older Chinese women with knee osteoarthritis. J Clin Rheumatol. 16(2):64-67.

[116] Field T (2011) Tai Chi research review. Complement Ther Clin Pract. 17(3):14-6.

[117] Uhlig T, Fongen C, Steen E, Christie A, Odegard S (2010) Exploring Tai Chi in rheumatoid arthritis: a quantitative and qualitative study. BMC Musculoskeletal Disorders; 5:11-43.

[118] Hall AM, Maher CG, Lam P, Ferreira M, Latimer J. Tai chi exercise for treatment of pain and disability in people with persistent low back pain: a randomized controlled trial. Arthritis Care Res (Hoboken). 2011 Nov;63(11):1576-83.

[119] Sova R (2005) Aquatic Training. In: Jones CJ, Rose DJ, editors. Physical Activity Instruction of Older Adults. Human Kinetics. pp. 248-261.

[120] Krein SL, Heisler M, Piette JD, Butchart A, Kerr EA (2007) Overcoming the influence of chronic pain on older patients' difficulty with recommended self-management activities. Gerontologist. 47(1):61-8.

[121] Resnick B, Avers D (2012) Motivation and Patient Education: Implications for Physical Therapist Practice. In: Guccione A, Wong R, Avers D, editors. Geriatric Physical Therapy. 3rd ed. Elsevier. pp. 183-206. 